\title{
COMPUTATIONAL STRATEGIES FOR LOWER BOUND BUCKLING LOADS OF WIND-LOADED SHELLS OF REVOLUTION
}

\author{
R. C. Jaca ${ }^{1}$, L. A. Godoy ${ }^{2}$
}

${ }^{1}$ Constructions Department, Facultad de Ingeniería de la Universidad Nacional del Comahue (corresponding-rossana.jaca@ fain.uncoma.edu.ar)

${ }^{2}$ Structures Department, FCEFyN de la Universidad Nacional de Córdoba

\begin{abstract}
Advances in computational methods to model the nonlinear elastic behavior of shell structures have generated new computer capabilities to solve complex industrial problems. Shell buckling involves a number of specific features not present in other structural forms, such as imperfection-sensitivity, load-sensitivity, mode interaction, and exchange of energy contributions along a nonlinear equilibrium path. However, rather than using increasingly complex tools, it is also desirable to have methods in which the physics of the problem is taken into account to set theoretically loaded models, which can be solved using simpler computational tools. The authors have recently investigated one such strategy, known as Reduced Stiffness Method, in which selected energy components are eroded as a consequence of mode interaction and imperfection-sensitivity. This physical interpretation allows the formulation as an eigenvalue problem, in which the critical loads are lower bounds to experiments or to nonlinear incremental analysis. This paper considers the computational implementation of a reduced stiffness approach to the buckling of axisymmetric shell structures under wind loads. The structural configurations of interest in this work are cylindrical storage tanks with a roof (either flat or conical roof), in which case the thickness is decreased from the bottom to the top of the cylindrical part. A reduced stiffness approach has been implemented in a finite element code for shells of revolution, in which stabilizing membrane components are eliminated on the assumption that they will be eroded due to imperfection-sensitivity and mode interaction. Several strategies are considered depending on the zone of the shell in which elimination of membrane components is made. The code is employed to estimate buckling loads and modes in tanks with various roof configurations. It is shown that there are two aspects that influence the results: first, the existence of zones with different stiffness in the structure, and second, a load with a circumferential variation. The present results are compared with geometrically nonlinear analysis including shape imperfections and with experimental results. The results indicate that a reduction in meridional and torsional membrane components applied on a zone of the shell leads to good results in terms of limit point loads and modes.
\end{abstract}

Keywords: Buckling, Elastic Stability, Lower Bounds, Shells, Wind. 


\section{INTRODUCCIÓN}

El pandeo de cáscaras es un problema complejo que requiere tener en cuenta aspectos particulares como lo es la sensibilidad ante imperfecciones geométricas o de cargas y el acoplamiento de modos. Este aspecto fue planteado por Donell [7] en la década del $30 \mathrm{y}$ en base a ello Croll y colaboradores [5] desarrollaron la metodología de rigidez reducida. La propuesta de Croll consiste en considerar que la contribución membranal a la energía elástica se erosiona por el acoplamiento de modos y la presencia de imperfecciones, conduciendo a una pérdida de la estabilidad con valores de cargas máximas resistidas menores que las obtenidas por un análisis de bifurcación. De esta manera planteando el problema de autovalores y autovectores donde se elimina la contribución membranal, se obtienen cargas que resultan límite inferior a las halladas por el análisis clásico y por un análisis no lineal geométrico con imperfecciones. Una ventaja de esta aproximación es que permite realizar una evaluación más simple del problema de pandeo mediante un análisis de autovalores modificado, sin necesidad de plantear análisis no lineal geométrico con imperfecciones de diferentes formas y amplitudes.

La teoría de límites inferiores basada los postulados establecidos por Croll [4] ha dado resultados efectivos para cáscaras de diferentes formas (aunque principalmente cilíndricas) de espesor constante y para cargas con simetría axial [6, 22, 24]. Aplicaciones más recientes de la metodología pusieron de manifiesto la necesidad de afrontar situaciones no tenidas en cuenta anteriormente. La motivación de este trabajo es extender la aplicación de la metodología de rigidez reducida para analizar el pandeo de cáscaras de espesor variable bajo la acción de cargas de viento, aplicada a tanques de almacenamiento de fluidos.

El análisis de cáscaras de espesor variable ha sido abordado por diferentes investigadores [15, 17, 18]. Las Recomendaciones Europeas [19] consideran el pandeo de cilindros de espesor variable mediante una transformación en un cilindro equivalente de espesor constante. Sosa et al. [20, 21] han aplicado la metodología de energía reducida bajo cargas de viento y presión uniforme mediante un programa de propósitos generales.

Trabajos anteriores realizados por los autores $[10,12]$ abordaron nuevas problemáticas asociadas a la implementación de la metodología de rigidez reducida relacionadas a cáscaras con diferentes espesores y/o cargas que no presentan simetría axial. El sistema estructural estudiado en este trabajo son tanques de almacenamiento de fluidos sometidos a la acción del viento, presentándose ambas características mencionadas anteriormente, ya que en el diseño del tanque basado en la norma API 650 [3] resultan cilindros con espesor escalonado, por presión variable en altura, y además, por viento, se producen deformadas con desplazamientos significativos a barlovento y prácticamente nulos a sotavento. La Figura 1 muestra un tanque con techo cónico en el que ambos problemas se presentan combinados, es decir distintos espesores en el cilindro, cuya deformada es producida por la acción del viento.

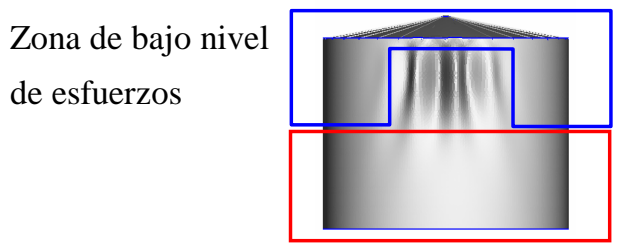

Zona de bajo nivel de deformación

Figura 1. Tanque con techo cónico con pandeo por acción del viento. 
Este trabajo se enfoca en analizar estrategias de implementación eficiente de la metodología de rigidez reducida para el sistema estructural mencionado anteriormente, que presenta aspectos no totalmente estudiados, con respecto a la manera más adecuada de realizar la eliminación de las componentes membranales.

La Sección 2 presenta el desarrollo teórico sobre el que se basan las diferentes estrategias y en la Sección 3 se presentan los casos estudiados. La explicación de la simulación numérica desarrollada se presenta en la Sección 4 y en la Sección 5 su implementación para un conjunto de tanques con techo cónico estudiados. En la Sección 6 se validan los resultados comparando los hallados al aplicar la metodología para un conjunto de tanques de techo plano con los obtenidos en ensayos en túnel de viento.

\section{FORMULACIÓN}

La modelación de la cáscara por Elementos Finitos plantea, a partir de la rigidez total del sistema, la condición de equilibrio para el cálculo de los estados precríticos a lo largo de la trayectoria fundamental lineal como:

$$
\begin{gathered}
\boldsymbol{K}_{0} \boldsymbol{a}+\lambda \boldsymbol{P}=0 . \\
\boldsymbol{K}_{0}=\int\left(\boldsymbol{B}_{0}^{T} \boldsymbol{D} \boldsymbol{B}_{0}\right) d A .
\end{gathered}
$$

donde $\mathbf{K}_{0}$ es la matriz de rigidez lineal de la cáscara, a es el vector desplazamientos nodales, $\lambda$ el parámetro de carga y $\mathbf{P}$ el vector de cargas escaladas mediante $\lambda$. Usando la notación común para un análisis de elementos finitos [23] la matriz de rigidez $\mathbf{K}_{0}$ se expresa en función de $\mathbf{B}_{0}$ que contiene las funciones de interpolación y la matriz constitutiva $\mathbf{D}$ obtenida a partir de las características del material. La condición de punto crítico resulta en el problema de autovalores planteado en (2):

$$
\begin{aligned}
& {\left[\boldsymbol{K}_{0}+\lambda^{C} \boldsymbol{K}^{G}\left(\boldsymbol{N}^{F}\right)\right] \boldsymbol{\Phi}=0 .} \\
& \boldsymbol{K}^{G}=\int\left(\boldsymbol{G}^{T} \boldsymbol{N}^{F} \boldsymbol{G}\right) d A .
\end{aligned}
$$

donde $\lambda^{\mathrm{C}}$ es la carga crítica del problema, y $\Phi$ es el autovector que indica la deformada asociada al modo crítico. La matriz $\mathbf{K}^{\mathrm{G}}$ tiene en cuenta las componentes no lineales del campo de deformaciones a través de la matriz $\mathbf{G}$ que vincula los giros con los desplazamientos y también incluye las tensiones iniciales en la trayectoria fundamental mediante $\mathbf{N}^{\mathrm{F}}$.

\subsection{Rigidez Reducida Selectiva en el Análisis de Bifurcación}

En la Figura 2 se considera en forma esquemática el dominio de la estructura dividido en dos sectores de características muy diferentes, en la Figura 2(a) la zona 2 presenta una mayor rigidez que la zona 1 proveniente, por ejemplo, de cáscaras con dos espesores diferentes $\mathrm{t}_{1}$ y $t_{2}>t_{1}$; o bien diferencias generadas por una zona inactiva por localización de modos (bajo nivel de desplazamientos y esfuerzos), como se muestra en la Figura 2(b). 


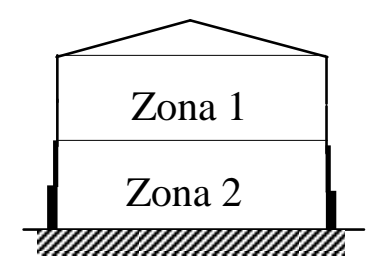

(a)

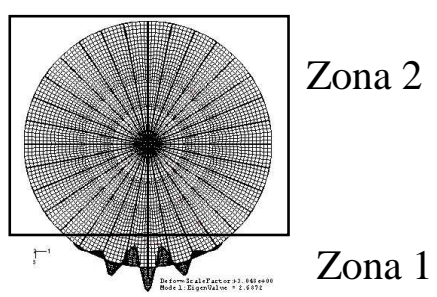

(b)

Figura 2. Esquema de estructura con zonas diferentes, (a) Influencia de rigidez,

(b) Influencia de esfuerzos.

La matriz de rigidez $\mathbf{K}_{0}$, para pequeños desplazamientos, contiene el aporte de la rigidez membranal $\mathbf{K}_{\mathrm{M}}$ y de la rigidez flexional $\mathbf{K}_{\mathrm{B}}$, donde se tiene en cuenta de manera separada la contribución de las zonas 1 y 2 consideradas, tal como lo pone en evidencia la Ec. (3).

$$
\boldsymbol{K}_{0}=\boldsymbol{K}_{M}+\boldsymbol{K}_{B} \quad \text { con } \quad \boldsymbol{K}_{M}=\left[\begin{array}{ll}
\boldsymbol{K}_{11}{ }^{M} & \boldsymbol{K}_{12}{ }^{M} \\
\boldsymbol{K}_{21}{ }^{M} & \boldsymbol{K}_{22}{ }^{M}
\end{array}\right] \quad, \quad \boldsymbol{K}_{B}=\left[\begin{array}{ll}
\boldsymbol{K}_{11}{ }^{B} & \boldsymbol{K}_{12}{ }^{B} \\
\boldsymbol{K}_{21}{ }^{B} & \boldsymbol{K}_{22}{ }^{B}
\end{array}\right] .
$$

Vinculado a la problemática puesta de manifiesto en la Figura 2(a), se puede comprobar que en estructuras de pared delgada la mayor rigidez es membranal y depende de $t$, mientras que la rigidez flexional resulta menor, dependiendo de $\mathrm{t}^{3}$. Por presentar la zona 2 una mayor rigidez producida por el espesor mayor se obtiene que $\mathbf{K}_{22}{ }^{\mathrm{M}} \gg \mathbf{K}_{11}{ }^{\mathrm{M}}$. En realidad, si se considera una teoría membranal, la rigidez flexional puede despreciarse por ser $\mathrm{t}<<1$ y la estructura sólo retendría la parte membranal. Bajo estas condiciones, el modo $\Phi$ solamente tiene componentes significativas en la zona de menor espesor $\Phi_{1}$ y en la zona más rígida resulta despreciable, es decir $\boldsymbol{\Phi}_{2} \approx 0$.

Además, basándose en las características mostradas en la Figura 2(b), se puede observar la influencia de la localización de modos que genera esfuerzos considerablemente mayores en una zona respecto a la otra, como en el caso de acción de viento. La zona a barlovento, zona 1, presenta esfuerzos mayores que en la zona a sotavento considerada como zona 2, entonces la matriz de estados precríticos $\mathbf{N}^{\mathrm{F}}$ tiende a ser mayor en la zona 1 afectando a $\mathbf{K}^{\mathrm{G}}$ y resultando $\mathbf{K}_{11}{ }^{\mathrm{G}}>>\mathbf{K}_{22}{ }^{\mathrm{G}}$ como también $\mathbf{K}_{11}{ }^{\mathrm{G}}>>\mathbf{K}_{12}{ }^{\mathrm{G}}$. Si no hubiera cambios relativos de rigidez entre las zonas por espesores diferentes, la matriz $\mathbf{K}_{0}$ no tendría cambios y esta concentración de esfuerzos solamente influiría en la condición de estado crítico, dada por la Ec. (2). De esta manera, el problema de autovalores con la contribución de las zonas 1 y 2 resulta:

$$
\begin{gathered}
{\left[\begin{array}{cc}
\boldsymbol{K}^{G}{ }_{11} & \boldsymbol{K}^{G}{ }_{12} \\
\boldsymbol{K}^{G}{ }_{21} & \boldsymbol{K}^{G}{ }_{22}
\end{array}\right] \text { y } \boldsymbol{\Phi}=\left[\begin{array}{l}
\boldsymbol{\Phi}_{1} \\
\boldsymbol{\Phi}_{2}
\end{array}\right] .} \\
\left\{\left[\begin{array}{cc}
\boldsymbol{K}_{11}{ }^{0} & \boldsymbol{K}_{12}{ }^{0} \\
\boldsymbol{K}_{21} 0 & \boldsymbol{K}_{22}{ }^{0}
\end{array}\right]+\lambda^{C}\left[\begin{array}{cc}
\boldsymbol{K}_{11}{ }^{G} & 0 \\
0 & 0
\end{array}\right]\right\}\left[\begin{array}{l}
\boldsymbol{\Phi}_{1} \\
\boldsymbol{\Phi}_{2}
\end{array}\right]=\left[\begin{array}{l}
0 \\
0
\end{array}\right] . \\
\Rightarrow\left[\begin{array}{c}
\left(\boldsymbol{K}_{11}{ }^{0}+\lambda^{C} \boldsymbol{K}_{11}{ }^{G}\right) \boldsymbol{\Phi}_{1}+\boldsymbol{K}_{12}{ }^{0} \boldsymbol{\Phi}_{2} \\
\boldsymbol{K}_{21}{ }^{0} \boldsymbol{\Phi}_{1}+\boldsymbol{K}_{22}{ }^{0} \boldsymbol{\Phi}_{2}
\end{array}\right]=\left[\begin{array}{l}
0 \\
0
\end{array}\right] . \\
\left(\boldsymbol{K}_{11}{ }^{0}+\lambda^{C} \boldsymbol{K}_{11}{ }^{G}\right) \boldsymbol{\Phi}_{1}+\boldsymbol{K}_{12}{ }^{0} \boldsymbol{\Phi}_{2}=0 .
\end{gathered}
$$


y el autovalor $\lambda^{\mathrm{C}}$ queda asociado a $\boldsymbol{\Phi}_{1}$ como se muestra en la Ec. (6), resultando $\boldsymbol{\Phi}_{2} \approx 0$ en las formas de modo obtenidas en el planteo con la rigidez completa.

Es decir, al realizar el análisis de bifurcación lineal con el aporte de todas las componentes de energía, membranal y flexional, la forma de modo tiene valores de desplazamientos significativos en la zona 1 , que corresponde a menor rigidez y/o a barlovento.

Al plantear la metodología de rigidez reducida, se elimina el aporte de las componentes membranales para obtener la respuesta de la cáscara con su menor capacidad, dada solamente por el aporte flexional, y obtener en el problema de autovalores una carga crítica y una forma de modo que representa un límite inferior. Si esta reducción se realiza en toda la cáscara, se genera una distorsión en la matriz $\mathbf{K}_{0}$ ya que $\mathbf{K}_{\mathrm{M}}$ desaparece por completo y se modifica el problema de autovalores de manera que $\Phi_{2}^{\prime} \neq 0$. Esto origina que se produzca una transferencia de deformaciones de la zona 1 a la zona 2 y pueden aparecer formas de modo asociadas con deformaciones por flexión que no son compatibles con las obtenidas en un análisis no lineal geométrico con seguimiento de trayectoria.

Un análisis alternativo fue abordado anteriormente por los autores [10] para cáscaras cilíndricas con diferentes espesores y presión uniforme, aplicando la rigidez reducida de manera selectiva, mediante la eliminación de las componentes membranales asociadas a la zona donde el modo crítico tiene desplazamientos significativos, es decir en la zona 1, resultando:

$$
\left\{\left[\begin{array}{cc}
0 & \boldsymbol{K}_{12}{ }^{M} \\
\boldsymbol{K}_{21}{ }^{M} & \boldsymbol{K}_{22}{ }^{M}
\end{array}\right]+\left[\begin{array}{cc}
\boldsymbol{K}_{11}{ }^{B} & \boldsymbol{K}_{12}{ }^{B} \\
\boldsymbol{K}_{21}{ }^{B} & \boldsymbol{K}_{22}{ }^{B}
\end{array}\right]+\lambda^{\prime \prime}\left[\begin{array}{ll}
\boldsymbol{K}_{11}^{G} & \boldsymbol{K}_{12}{ }^{G} \\
\boldsymbol{K}_{21}{ }^{G} & \boldsymbol{K}_{22}{ }^{G}
\end{array}\right]\right\}\left[\begin{array}{l}
\boldsymbol{\Phi}_{1}^{\prime \prime} \\
\boldsymbol{\Phi}_{2}^{\prime \prime}
\end{array}\right]=\left[\begin{array}{l}
0 \\
0
\end{array}\right] .
$$

o bien:

$$
\left\{\left[\begin{array}{cc}
0 & 0 \\
0 & \boldsymbol{K}_{22}{ }^{M}
\end{array}\right]+\left[\begin{array}{cc}
\boldsymbol{K}_{11}{ }^{B} & \boldsymbol{K}_{12}{ }^{B} \\
\boldsymbol{K}_{21}{ }^{B} & \boldsymbol{K}_{22}{ }^{B}
\end{array}\right]+\lambda^{\prime \prime \prime}\left[\begin{array}{ll}
\boldsymbol{K}_{11}{ }^{G} & \boldsymbol{K}_{12}{ }^{G} \\
\boldsymbol{K}_{21}{ }^{G} & \boldsymbol{K}_{22}{ }^{G}
\end{array}\right]\right\}\left[\begin{array}{l}
\boldsymbol{\Phi}_{1}^{\prime \prime \prime} \mid \\
\boldsymbol{\Phi}_{2}^{\prime \prime \prime}
\end{array}\right]=\left[\begin{array}{l}
0 \\
0
\end{array}\right] .
$$

Como $\mathbf{K}_{22}{ }^{\mathrm{M}}$ no se ha perdido y es la mayor rigidez original del sistema, entonces no habrá transferencia de deformaciones hacia la zona 2 y continuará siendo $\Phi_{2}{ }^{\prime \prime} \cong 0$. Con esta modificación se obtuvieron buenos resultados para determinar límites inferiores a las cargas de pandeo y también se pudieron aproximar de manera adecuada los modos asociados. Se puede observar que en este caso de rigideces diferentes por espesor variable, la matriz de carga-geometría $\mathbf{K}^{\mathrm{G}}$ se mantiene igual que en el planteo con la versión completa de la energía potencial.

Para abordar la problemática de los modos localizados que genera la carga de viento, se deben tener en cuenta los cambios en la condición de punto crítico que aparecen por la modificación de la matriz $\mathbf{K}^{\mathrm{G}}$, como lo expresa la Ec. (6). Al plantear la metodología de rigidez reducida, se elimina la componente membranal y entonces en la Ec.(6) también se ve afectada la matriz $\mathbf{K}^{\mathrm{M}}$, influyendo en los resultados del análisis de autovalores y autovectores. Esto se produce ya que cuando se elimina el aporte membranal $\left(\mathbf{K}^{\mathrm{M}}\right)$ en toda la cáscara se modifican las relaciones entre términos de zonas 1 y 2 en la matriz de rigidez $\mathbf{K}_{0}$ original del sistema y otra vez resulta en la forma de modo que $\Phi_{2} \neq 0$, apareciendo nuevamente una transferencia de deformaciones a la zona a sotavento que no concuerda con las evidencias de daño.

El planteo alternativo de una reducción selectiva de las componentes membranales, 
hace posible que $\mathbf{K}_{22}{ }^{\mathrm{M}}$ en $\mathbf{K}_{0}$ no desaparezca y se elimine solamente el aporte membranal en la zona 1 que no presenta prácticamente esfuerzos y desplazamientos, resultando nuevamente $\Phi_{2} \approx 0$ con formas de modo compatibles con lo observado en el análisis no lineal geométrico.

\section{DESCRIPCIÓN DE LOS MODELOS Y CARGAS}

Los estudios realizados se refieren a dos conjuntos de tanques de diferentes características, unos con techo cónico y otros de techo plano fijo de diversas relaciones geométricas, bajo presión de viento. Los modelos con techo cónico ya que fueron estudiados anteriormente bajo viento por Sosa et al. [20,21] y por Jaca et al. [10] bajo presión uniforme, lo que permite ajustar la metodología cubriendo un rango de tanques típicos encontrados en el campo. El otro grupo de tanques analizados han sido ensayados en túnel de viento por Ressinger y Greiner [15] y permiten una validación de la metodología implementada.

Todos los tanques de techo cónico tienen el mismo diámetro de $30.48 \mathrm{~m}$ con diferente altura resultando relaciones de aspecto altura del cilindro-diámetro (H/D) que varía de 0.24 a 0.95. El espesor de la cáscara cilíndrica, reducido gradualmente mediante el método de un pie especificado en la norma API 650, está presentado en la Tabla 1. El techo de todos los modelos es cónico y fijo, reforzado por 32 vigas con una pendiente de techo de $3 / 16$ " y un espesor de $0.007 \mathrm{~m}$, el cilindro se considera empotrado en la base. El material constitutivo es acero con módulo de elasticidad de $\mathrm{E}=2.06 \mathrm{GPa}$ y coeficiente de Poisson 0.3.

Tabla 1. Espesores adoptados para cada modelo.

\begin{tabular}{|c|c|c|c|c|c|c|}
\hline \multirow{2}{*}{ Virola } & MC1 & MC2 & MC3 & MC4 & MC5 & MC6 \\
\hline & \multicolumn{6}{|c|}{$\mathrm{t}_{\text {diseño }}[\mathrm{m}] \mathrm{t}_{\text {diseño }}[\mathrm{m}] \mathrm{t}_{\text {diseño }}[\mathrm{m}] \mathrm{t}$} \\
\hline 1 & 0.0095 & 0.0127 & 0.0175 & 0.0206 & 0.0254 & 0.0286 \\
\hline 2 & 0.0079 & 0.0111 & 0.0159 & 0.0175 & 0.0222 & 0.0254 \\
\hline 3 & 0.0079 & 0.0079 & 0.0127 & 0.0159 & 0.0206 & 0.0254 \\
\hline 4 & & 0.0079 & 0.0111 & 0.0127 & 0.0175 & 0.0222 \\
\hline 5 & & 0.0079 & 0.0095 & 0.0111 & 0.0159 & 0.0206 \\
\hline 6 & & & 0.0079 & 0.0079 & 0.0127 & 0.0191 \\
\hline 7 & & & 0.0079 & 0.0079 & 0.0111 & 0.0159 \\
\hline 8 & & & & 0.0079 & 0.0079 & 0.0127 \\
\hline 9 & & & & & 0.0079 & 0.0111 \\
\hline 10 & & & & & 0.0079 & 0.0079 \\
\hline 11 & & & & & & 0.0079 \\
\hline 12 & & & & & & 0.0079 \\
\hline
\end{tabular}

Los tanques ensayados en túnel de viento forman un conjunto de seis modelos compuestos por una cáscara cilíndrica de espesor constante con techo plano. El material constitutivo es aluminio, con módulo de elasticidad de $70000 \mathrm{~Pa}$ y coeficiente de Poisson (v) de 0.347. La Tabla 2 presenta las dimensiones de los modelos estudiados y las correspondientes relaciones adimensionales asociados a la cáscara cilíndrica, donde $\mathrm{L}$ es la altura del tanque, $\mathrm{r}$ es su radio y $\mathrm{Z}$ el parámetro adimensional de Batdorf dado por la Ec. 9. 


$$
Z=\frac{L^{2}}{r t} \sqrt{1-v^{2}}
$$

Tabla 2. Dimensiones de los modelos de techo plano ensayados en túnel de viento.

\begin{tabular}{|c|c|c|c|c|c|c|}
\hline Modelo & $\begin{array}{c}\mathrm{r} \\
{[\mathrm{mm}]}\end{array}$ & $\begin{array}{c}\mathrm{L} \\
{[\mathrm{mm}]}\end{array}$ & $\begin{array}{c}\mathrm{t} \\
{[\mathrm{mm}]}\end{array}$ & $\mathrm{L} / \mathrm{r}$ & $r / t$ & Z \\
\hline MR1 & \multirow{3}{*}{220} & 440 & 0.105 & 2 & \multirow{3}{*}{2095} & 7860 \\
\hline MR2 & & 320 & 0.105 & 1.46 & & 4157 \\
\hline MR3 & & 180 & 0.100 & 0.82 & & 1381 \\
\hline MR4 & \multirow{3}{*}{310} & 320 & 0.105 & 1.03 & \multirow{3}{*}{2952} & 3037 \\
\hline MR5 & & 250 & 0.105 & 0.81 & & 1800 \\
\hline MR6 & & 180 & 0.105 & 0.58 & & 933 \\
\hline
\end{tabular}

La variación espacial de presiones de viento en la parte cilíndrica del tanque se supone constante en altura y variable alrededor de la circunferencia, como en otros trabajos de investigación en el campo. Para las presiones que actúan en el techo se considera una distribución de presiones obtenidas en ensayos de túnel de viento por Macdonald et al. [13], que son similares a las presentadas por Portela y Godoy [14]. La presión máxima sobre la cáscara cilíndrica es de $1 \mathrm{KPa}$ actuando en el meridiano a barlovento. Para el análisis de estabilidad, los valores de las presiones que actúan en la cáscara completa se aumentan usando el parámetro de carga $(\lambda)$. La distribución de presión de viento adoptada sobre las paredes cilíndricas del tanque fue la correspondiente a la recomendación ACI-ASCE [2]:

$$
p=\lambda \sum_{i=1}^{n} c_{i} \cos (i \theta)
$$

con los siguientes coeficientes de Fourier: $\mathrm{c}_{0}=0.2765, \mathrm{c}_{1}=-0.3419, \mathrm{c}_{2}=-0.5418, \mathrm{c}_{3}=-0.3872$, $\mathrm{c}_{4}=-0.0525, \mathrm{c}_{5}=0.0771, \mathrm{c}_{6}=0.0039, \mathrm{c}_{7}=-0.0341$, donde $\mathrm{p}$ es la presión externa del viento $\mathrm{y}$ $\theta$ es el ángulo con respecto a la dirección del viento. La Figura 3(a) muestra la distribución de presión usada en el techo cónico y la Figura 3(b) muestra la distribución de presión de viento adoptada alrededor de la circunferencia de la cáscara cilíndrica.

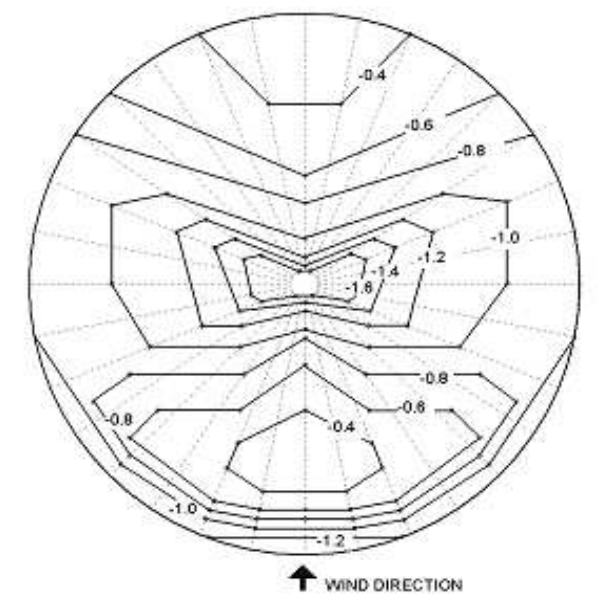

(a)

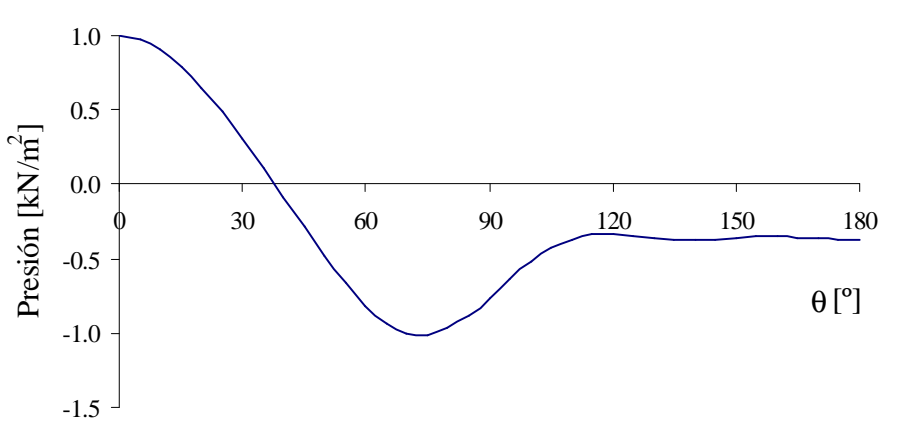

(b)

Figura 3. Distribución de presión de viento (a) en el techo cónico;

(b) alrededor del cilindro. 


\section{MODELO COMPUTACIONAL}

Todos los tanques fueron modelados por Elementos Finitos, mediante el programa de propósitos generales ABAQUS [1] usando elementos de cáscaras triangulares y cuadrangulares y también por el programa de propósitos específicos ALREF [8], usando elementos semianalíticos que discretizan el meridiano con elementos curvos unidimensionales. En ambos casos se utilizan mallas lo suficientemente densas para asegurar la convergencia.

Las cargas de viento en ABAQUS se incorporan como cargas de superficie actuando sobre los elementos de cáscaras según las distribuciones mostradas en la Figura 3, tanto para el techo cónico como para la cáscara cilíndrica. Debido a que en el código ALREF se modela solamente el meridiano, la distribución de carga sobre el techo se representa a través de series de Fourier para extenderla en toda la superficie según lo expresado en la Ec. (10). Para ello, la carga sobre el techo se divide en tres tramos diferentes para tener en cuenta la variación que se presenta en el meridiano, como se muestra en la Figura 4, resultando los coeficientes indicados en la Tabla 3.

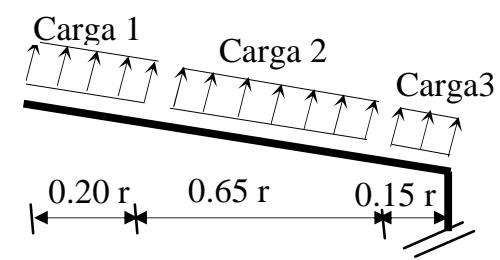

Figura 4. Cargas de viento sobre el techo cónico.

Tabla 3. Coeficientes para carga de viento en el techo cónico.

\begin{tabular}{crrcccccc}
\hline Carga & $\mathrm{C}_{0}$ & $\mathrm{C}_{1}$ & $\mathrm{C}_{2}$ & $\mathrm{C}_{3}$ & $\mathrm{C}_{4}$ & $\mathrm{C}_{5}$ & $\mathrm{C}_{6}$ & $\mathrm{C}_{7}$ \\
\hline \hline 1 & 1.419 & 0.063 & -0.203 & 0.03 & -0.026 & 0.008 & -0.0016 & 0.0009 \\
\hline 2 & 0.607 & 0.034 & -0.172 & -0.043 & 0.031 & -0.011 & 0.015 & -0.013 \\
\hline 3 & 0.755 & 0.035 & -0.036 & -0.008 & 0.021 & -0.020 & -0.0005 & 0.015 \\
\hline
\end{tabular}

La implementación de la metodología de rigidez reducida mediante Elementos Finitos se realiza usando el código de propósitos específicos ALREF, en el cual se realizaron modificaciones para incorporar la evaluación de las diferentes componentes de energía y selectivamente reducir alguna de estas contribuciones. ALREF es un código orientado al análisis de tensiones y estabilidad de cáscaras de revolución bajo cargas axilsimétricas y no axilsimétricas. El programa utiliza un elemento curvo unidimensional que discretiza la cáscara en al sentido del meridiano y representa el comportamiento del paralelo mediante series de Fourier. El elemento tiene tres nodos: uno en cada extremo con cuatro incógnitas (desplazamientos $\mathrm{u}, \mathrm{v}$, w y giro $\partial u / \partial s)$ y un nudo central con seis incógnitas $(u, \partial u / \partial s, v, \partial v / \partial s, w, \partial w / \partial s)$, donde u es el desplazamiento en el sentido del meridiano, v es el desplazamiento en el sentido del paralelo y w es el desplazamiento en el sentido de la normal a la cáscara. La geometría se define por una interpolación cúbica en base a las coordenadas y tangentes en cada nodo, mientras que el campo de desplazamientos se aproxima por funciones cúbicas para el desplazamiento en el plano de la cáscara y quínticas para el desplazamiento fuera del plano.

Para cargas no axilsimétricas como las de viento, hay un acoplamiento de modos en el cálculo de autovalores por lo que es necesario especificar cuáles y cuántas armónicas serán 
utilizadas en el cálculo de las componentes de energía asociadas al punto crítico y para el planteo del problema de autovalores con energía completa o reducida. Como criterio de selección para determinar cuáles armónicas usar se considera el criterio conocido como del meridiano con mayores tensiones, usando una cierta cantidad de armónicas consecutivas que individualmente arrojan las menores cargas críticas para carga radial unitaria.

En trabajos anteriores de los autores $[9,10]$ se ha implementado la metodología de rigidez reducida para tanques de techo flotante bajo viento o para tanques de techo fijo o flotante con presión uniforme, donde se describe más en detalle la implementación de la misma.

Los resultados de la aplicación de la metodología son validados con resultados experimentales o con resultados obtenidos mediante análisis no lineal geométrico considerando imperfecciones geométricas, realizado mediante el método de Riks [16] implementado en ABAQUS.

\section{TANQUES CON TECHO CÓNICO}

\subsection{Análisis de Bifurcación Lineal}

La Figura 5 muestra el primer modo crítico para todos los modelos, obtenido mediante el código ABAQUS. Se observa en esta figura que, en todos los modelos, la zona pandeada se concentra en las virolas con menor espesor en el cilindro. También se puede observar que las formas de modo correspondientes al conjunto de tanques analizados tienen desplazamientos concentrados en la zona de presiones positivas a barlovento. Esta respuesta también se presenta en tanques con evidencia de pandeo por viento y en las deformadas asociadas a estados poscríticos avanzados. Esta característica de la respuesta, ya presente en el análisis de bifurcación lineal, sirve de guía para decidir donde realizar la eliminación selectiva de las componentes membranales.

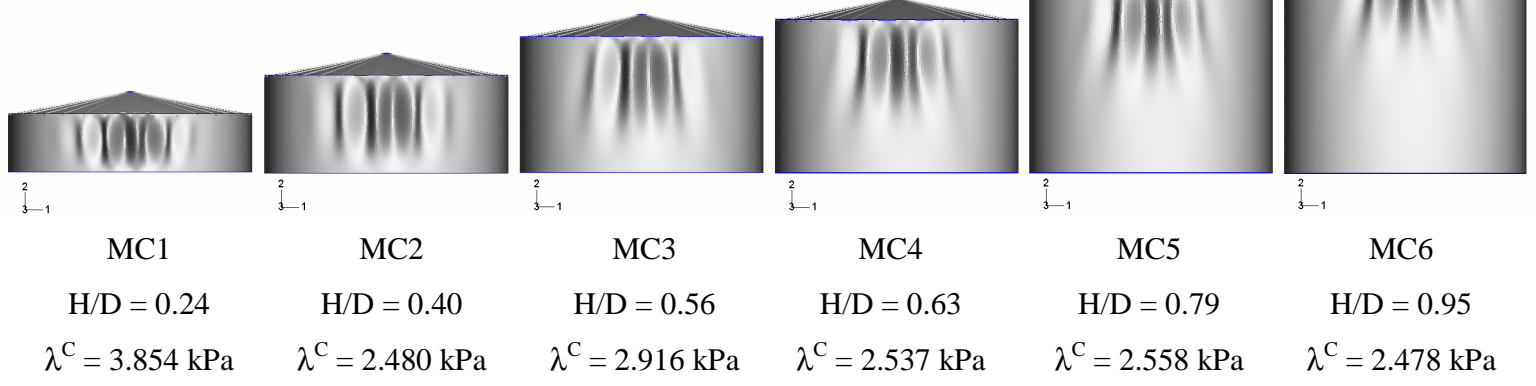

Figura 5. Primer modo de pandeo de los cinco modelos estudiados.

Los resultados obtenidos con el código ALREF son consistentes con los hallados con ABAQUS, en la Tabla 4 se muestran los valores de las cargas críticas para todos los modelos estudiados y en la Figura 6, la forma de modo hallada para el modelo identificado como MC4 que resulta similar a la hallada para los restantes tanques. 


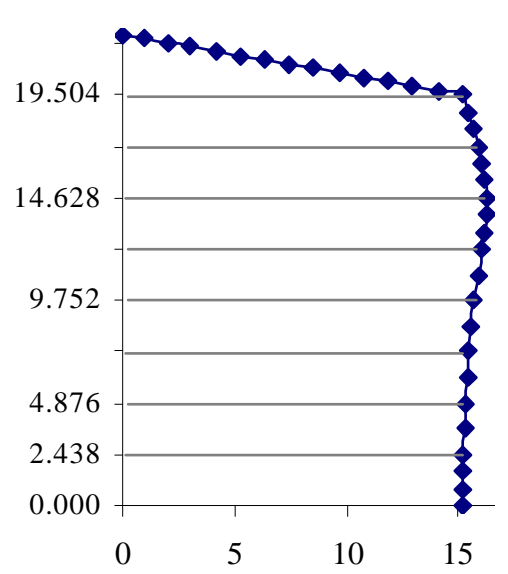

(a)

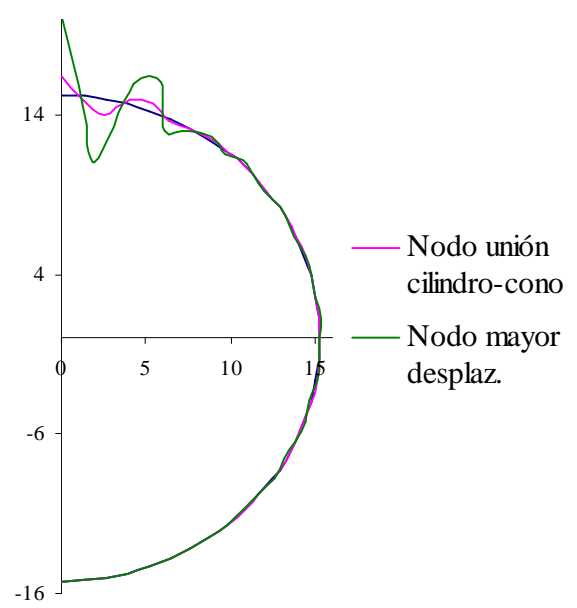

(b)

Figura 6. Forma de modo del modelo MC4 $\left(\lambda^{\mathrm{C}}=2.512 \mathrm{kPa}\right)$, (a) en el meridiano, (b) en el paralelo.

Tabla 4. Cargas críticas halladas con código ALREF.

\begin{tabular}{lllllll} 
& MC1 & MC2 & MC3 & MC4 & MC5 & MC6 \\
\hline$\lambda^{\mathrm{C}}[\mathrm{kPa}]$ & 3.885 & 2.551 & 2.921 & 2.512 & 2.542 & 2.497 \\
\hline
\end{tabular}

\subsection{Análisis No Lineal Geométrico con Imperfecciones}

Un análisis elástico no lineal geométrico considerando sensibilidad ante imperfecciones (GNIA, como se denomina en Rotter y Schmidt [19]) se llevó a cabo para comparar los límites inferiores con los obtenidos por la metodología de rigidez reducida. En todos los modelos se consideran imperfecciones de pequeña amplitud según la forma del primer autovector. Las amplitudes de las imperfecciones varían de $0.10 \mathrm{t}_{\min }$ a $1.0 \mathrm{t}_{\min }$ donde el $\mathrm{t}_{\min }$ es el menor espesor de la cáscara cilíndrica en cada caso. Para cada imperfección se calcularon las trayectorias de equilibrio no lineal usando el método de Riks [16] mediante ABAQUS, como una manera de exponer el comportamiento poscrítico.
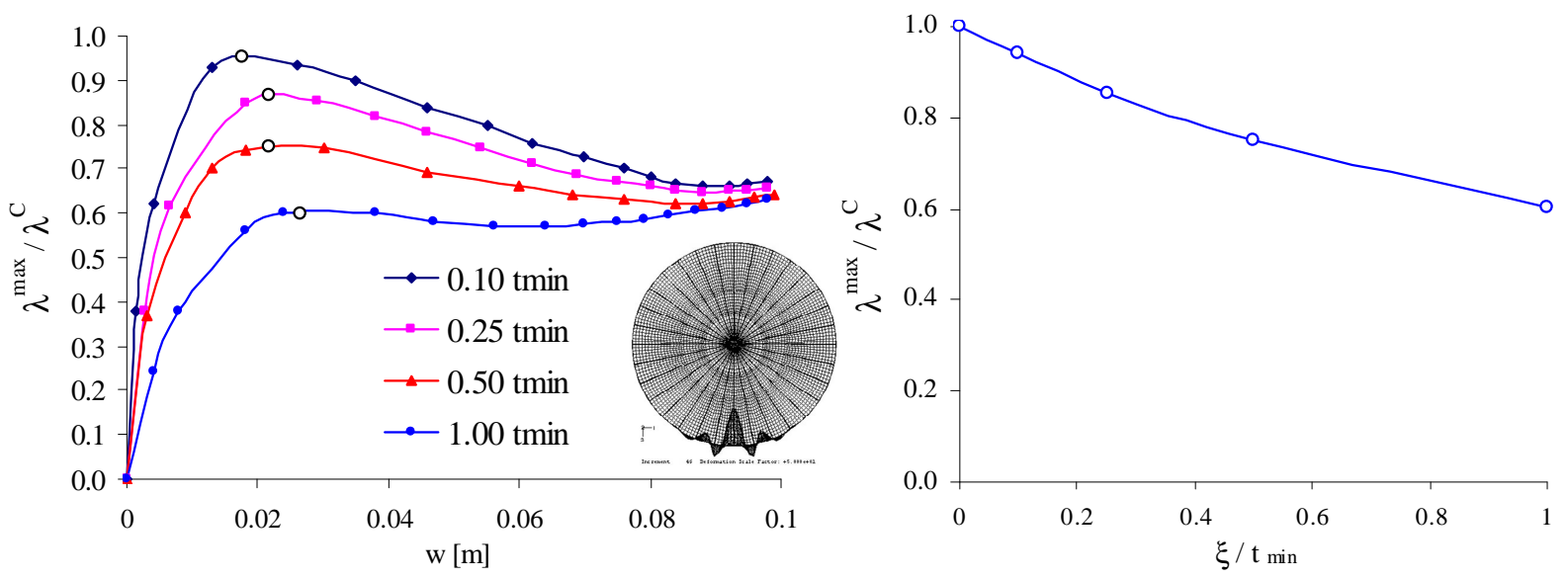

Figura 7. Trayectorias de equilibrio no lineal y curvas de sensibilidad ante imperfecciones, $\operatorname{MC} 4\left(\mathrm{H} / \mathrm{D}=0.63 ; \lambda^{\mathrm{C}}=2.537 \mathrm{kPa}\right.$, (Figura 5)).

El comportamiento de estas estructuras bajo acción de viento es similar para las distintas relaciones geométricas consideradas. Para imperfecciones de pequeña amplitud, por ejem- 
plo $0.10 \mathrm{t}_{\mathrm{min}}$, la trayectoria poscrítica no lineal es inestable. La estructura llega a un valor de carga máxima con pequeños desplazamientos, posteriormente no puede tomar más cargas y se presentan grandes deformaciones en la respuesta. A medida que aumenta la magnitud de la imperfección la carga máxima que toma la estructura va disminuyendo, para una amplitud de imperfección del orden del espesor de la cáscara cambia la respuesta de la estructura ya que la curva se hace monótona creciente y sigue tomando cargas adicionales con grandes desplazamientos. Este nivel de imperfección establece un límite inferior a las cargas que la estructura puede soportar con desplazamientos pequeños de su forma original. Después de ese límite, la estructura deformada se comporta de una manera diferente.

La Figura 7 muestra las trayectorias de equilibrio no lineales del modelo MC4 para los niveles mencionados de imperfecciones. La curva de sensibilidad ante imperfecciones mostrada en el lado derecho de la Figura 7 presenta la carga crítica normalizada $\lambda / \lambda_{\max }$ para cada nivel de imperfección en función de la amplitud de imperfección adimensionalizada $\xi / \mathrm{t}_{\min }$. El factor de reducción $\eta=\lambda_{\max } / \lambda^{\mathrm{C}}$ es directamente comparable con los resultados obtenidos usando el método de rigidez reducida. Para todos los modelos, el factor de reducción así calculado toma un valor de 0.6 aproximadamente.

La sensibilidad ante imperfecciones es prácticamente la misma para todos los casos estudiados, esto puede ser debido a que la zona con evidencia de pandeo está concentrada en la parte superior de la cáscara cilíndrica, donde los espesores son menores. Al calcular los espesores en esa zona próxima al techo resulta el espesor mínimo para todos los casos. Este hecho avala el uso del cilindro sustituto propuesto por Resinger y Greiner [15] como una manera de hallar las cargas críticas en el caso de cilindros de diferentes espesores.

\subsection{Análisis de Rigidez Reducida}

La modelación se realiza mediante el código ALREF, para la cáscara cilíndrica se densifican los elementos en la zona que presenta mayores desplazamientos en el modo crítico dividiendo en partes iguales los tramos de igual espesor. Se adopta un espesor del techo cónico de $0.015 \mathrm{~m}$, mayor que el real ya que las vigas rigidizadoras no fueron incluidas en el modelo.

Debido al acoplamiento de modos en el cálculo de autovalores, que se produce para cargas no axilsimétricas como las de viento, es necesario especificar el rango de armónicas que deben ser usadas en el problema de autovalores, tanto para calcular la carga crítica como para la evaluación de las componentes de energía. Por ello en una primera etapa se analizan los modelos bajo la acción de carga radial uniforme unitaria, en cuyo caso el cálculo resulta desacoplado para las distintas armónicas. Estos resultados ya fueron presentados en [10, 11] para el análisis de carga uniforme, en este caso son necesarios nuevamente para identificar el número de armónicas a considerar en la evaluación del problema bajo cargas no axilsimétricas.

La Figura 8(a) muestra la distribución de cargas críticas para todos los modelos según el modo con j ondas en la dirección circunferencial y en la Figura 8(b) se presentan las componentes de energía para el modelo MC4. El espectro de cargas críticas no presenta un único mínimo, ya que se trata de estructuras compuestas por dos cáscaras simples, por esa razón se 
considera un rango de armónicas que incluya ambos mínimos (valores de j entre 5 y 35) en el cálculo de autovalores y autovectores y de las correspondientes componentes de energía. Se realiza la evaluación para cada armónica de las componentes de energía membranal y flexional, usando las deformaciones y tensiones correspondientes. La energía membranal (Um) tiene sus componentes meridional (Umss), circunferencial (Umtt) y torsional (Umst); mientras que la energía flexional (Ub) está formada por el aporte meridional (Ubss), circunferencial (Ubtt) y torsional (Ubst).

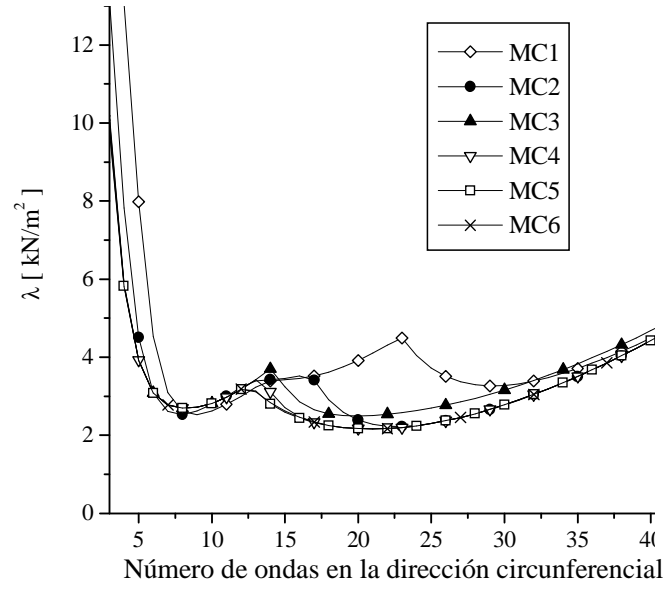

(a)

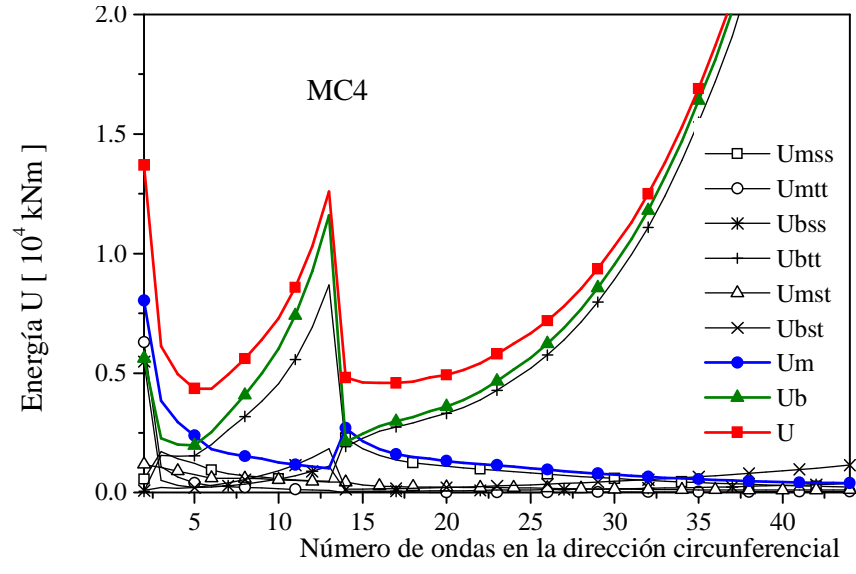

(b)

Figura 8. Modelación con ALREF, (a) Espectro de cargas críticas para los diferentes modelos, (b) Distribución de componentes de energía para el modelo MC4.

Por la interacción de ambas cáscaras la curva de energía total tampoco presenta un único mínimo, pero se observa que para modos con longitud de onda largos hay predominio de componentes membranales y para modos con longitud de onda cortos prevalece la contribución flexional, característica que se repite para este tipo de cáscaras. Es decir que, en general, el comportamiento es similar al observado cuando se analiza solamente el cilindro, salvo por esa zona intermedia en la que influye la rigidez relativa de una cáscara respecto a otra.

Al considerar la carga de viento se toma un conjunto de armónicas consecutivas (entre 20 y 30 armónicas), obteniéndose las cargas críticas mostradas en la Tabla 4 en el análisis de autovalores y autovectores. Para cada modelo se hace una evaluación de las componentes de energía en el punto crítico resultando que, en general, la energía membranal está compuesta por un $83 \%$ de aporte meridional (Umss) y un $17 \%$ de aporte torsional (Umst) con contribución casi nula o negativa de la componente circunferencial (Umtt). Este análisis es indicativo de qué componentes membranales eliminar en el planteo del problema de autovalores y autovectores. Dado que la componente circunferencial (Umtt) es desestabilizante o muy poco significativa, se suprime el aporte meridional y torsional en el análisis de energía reducida. De esta manera, en el cálculo de $\mathrm{B}_{0}$ que relaciona el campo de desplazamientos con el de deformaciones, se anulan los coeficientes que aportan a $\varepsilon_{\mathrm{ss}} \mathrm{y} \varepsilon_{\mathrm{st}}$, respectivamente, en forma acumulativa para evaluar cómo varían las cargas críticas al eliminar las componentes de energía membranal asociadas a esas deformaciones específicas. El cálculo de $\mathrm{K}^{\mathrm{G}}$, que relaciona los giros con los desplazamientos, se realiza con la matriz $\mathrm{N}^{\mathrm{F}}$ obtenida en base al análisis estático para el estado precrítico. De esta manera se obtienen las cargas críticas con la energía reducida $\left(\lambda^{*}\right)$, eliminando las componentes estabilizantes tanto en el techo cónico como en la envol- 
vente cilíndrica. La Tabla 5 muestra los valores hallados eliminando solamente la componente circunferencial y también los resultados obtenidos anulando las dos componentes membranales que son estabilizantes. En ambos casos, se indica el factor de reducción $\eta$ planteado como la relación entre $\lambda^{*}$ y $\lambda^{\mathrm{C}}$.

Tabla 5. Cargas críticas con rigidez reducida en toda la estructura.

\begin{tabular}{|c|c|c|c|c|c|}
\cline { 2 - 6 } \multicolumn{1}{c|}{} & Um completa & \multicolumn{2}{c|}{ Um-Umss } & \multicolumn{2}{c|}{ Um-Umss-Umst } \\
\cline { 2 - 6 } \multicolumn{1}{c|}{} & $\lambda^{\mathrm{C}}[\mathrm{kPa}]$ & $\lambda^{*}[\mathrm{kPa}]$ & $\eta$ & $\lambda^{*}[\mathrm{kPa}]$ & $\eta$ \\
\hline MC1 & 3.885 & 1.942 & 0.50 & 1.994 & 0.51 \\
\hline MC2 & 2.551 & 1.485 & 0.58 & 1.320 & 0.52 \\
\hline MC3 & 2.921 & 1.772 & 0.61 & 1.612 & 0.55 \\
\hline MC4 & 2.512 & 1.593 & 0.63 & 1.443 & 0.57 \\
\hline MC5 & 2.542 & 1.649 & 0.64 & 1.358 & 0.53 \\
\hline MC6 & 2.497 & 1.730 & 0.69 & 1.479 & 0.59 \\
\hline
\end{tabular}

En la Tabla 5 se puede observar que la reducción de carga crítica obtenida eliminando las dos componentes membranales estabilizantes establecen un límite inferior para las cargas críticas, si se lo compara con los obtenidos en el procedimiento de seguimiento de trayectoria donde se obtienen valores de 0.6 para el factor de reducción. Al eliminar solamente Umss se obtienen factores de reducción con un rango de variación mucho más amplio que lo obtenido con análisis no lineal geométrico y no resultan valores límite inferior en algunos casos. Por esta razón, a continuación se mantiene el criterio de eliminar ambas componentes.

Estas cargas críticas fueron obtenidas eliminando las contribuciones membranales en toda la cáscara, incluyendo el techo, y se puede observar un comportamiento diferente al observado en el análisis no lineal en cuanto a que los factores de reducción no son similares para todos los casos analizados sino que van aumentando a medida que aumenta la relación H/D. Además, al observar los modos asociados a estas cargas críticas, resulta que no son compatibles con las evidencias de daño ni con lo observado en el análisis no lineal en el que se obtiene daño a barlovento y en la zona próxima al techo. Las diferencias aparecen tanto en el meridiano, con deformaciones significativas en la zona de mayores espesores, como en el paralelo, presentándose componentes de desplazamiento importantes fuera de la zona a barlovento. Estos resultados confirman la transferencia de deformaciones que se produce tanto por zonas de diferente rigidez por distintos espesores como por la presencia de zonas con mayores esfuerzos por mayores desplazamientos.

\subsection{Rigidez Reducida Selectiva sobre el cilindro}

Un primer intento de reducción selectiva para tener en cuenta el efecto de pandeo localizado y evitar la transferencia de desplazamientos a zonas mas rígidas, es no eliminar las componentes membranales en el techo ya que posee una rigidez mucho mayor que el cilindro por la existencia de las vigas rigidizadoras. En la Tabla 6 se muestran los valores de cargas críticas $\left(\lambda^{*}\right)$ obtenidas reduciendo las componentes membranales meridionales y torsionales (Um-Umss-Umst) en toda la cáscara cilíndrica y los factores de reducción ( $\left.\eta^{*}\right)$ planteados como la relación con la carga crítica proveniente del análisis de bifurcación clásico $\left(\lambda^{\mathrm{C}}\right)$. Se 
puede notar que los factores de reducción hallados con esta reducción sectorizada disminuyen con respecto a los hallados con reducción en toda la cáscara y en general llegan a valores del orden del hallado para el tanque $\mathrm{MC}$, confirmando el hecho que el comportamiento de todos los modelos es semejante debido a que el modo de pandeo es localizado.

Tabla 6. Cargas críticas con rigidez reducida en cilindro, bajo cargas de viento.

\begin{tabular}{|c|c|c|c|}
\cline { 2 - 4 } \multicolumn{1}{c|}{} & $\begin{array}{c}\text { Um } \\
\text { completa }\end{array}$ & \multicolumn{2}{c|}{$\begin{array}{c}\text { Um-Umss-Umst } \\
\text { (en todo el cilindro) }\end{array}$} \\
\cline { 2 - 4 } \multicolumn{1}{c|}{} & $\lambda^{\mathrm{C}}[\mathrm{kPa}]$ & $\lambda^{*}[\mathrm{kPa}]$ & $\eta^{*}$ \\
\hline MC1 & 3.885 & 1.998 & 0.51 \\
\hline MC2 & 2.551 & 1.308 & 0.51 \\
\hline MC3 & 2.921 & 1.519 & 0.50 \\
\hline MC4 & 2.512 & 1.357 & 0.54 \\
\hline MC5 & 2.542 & 1.231 & 0.48 \\
\hline MC6 & 2.497 & 1.400 & 0.56 \\
\hline
\end{tabular}

MC6

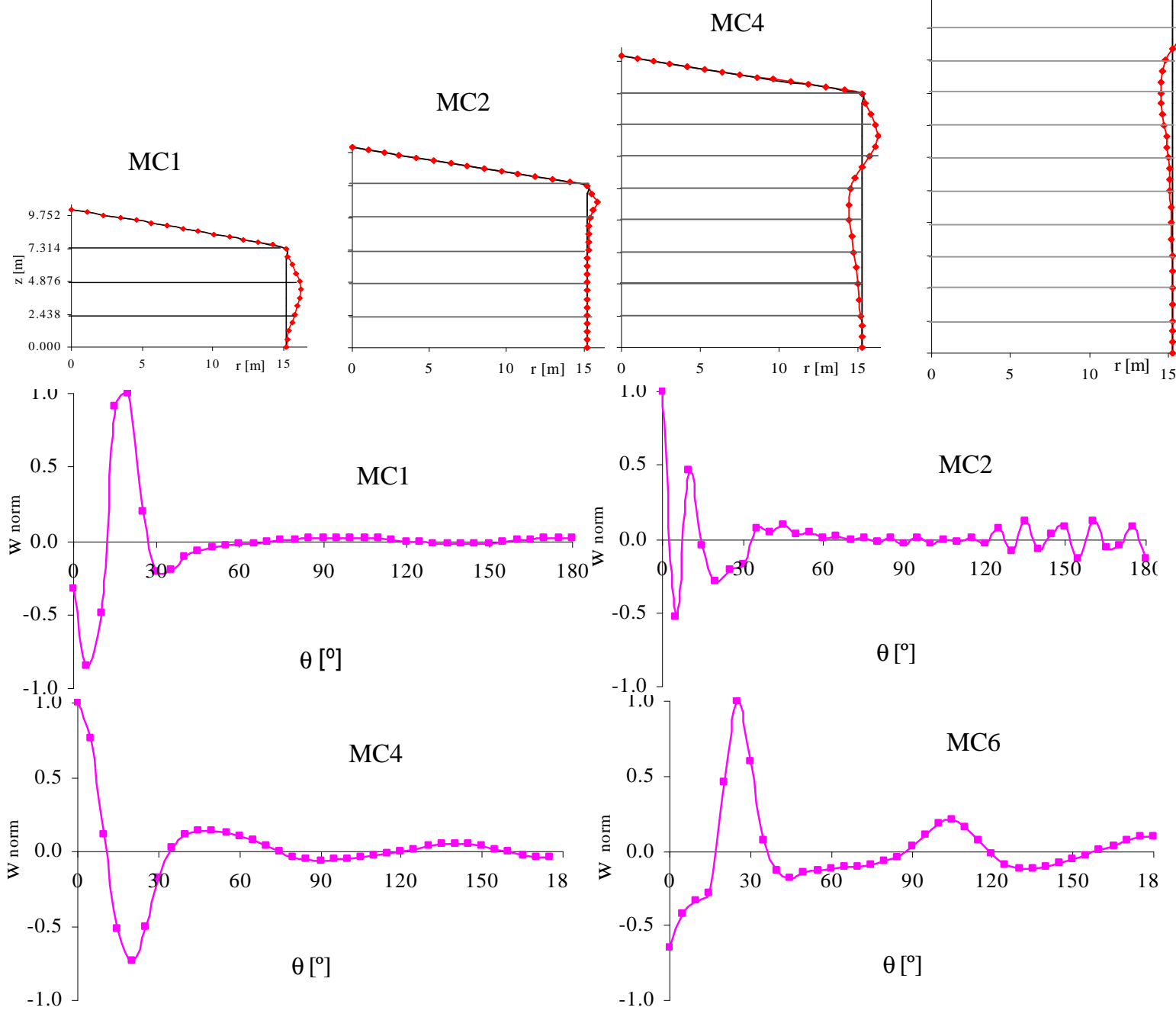

Figura 9. Formas de modo obtenidas con eliminación homogénea en todo el cilindro de las componentes membranales meridional y torsional. 
La Figura 9 muestra las formas de modo para los modelos denominados MC1, MC2, MC4 y MC6, ya sea en el meridiano a barlovento ubicado sobre el eje de simetría o en el paralelo correspondiente al nodo de mayor desplazamiento, cuando se plantea la eliminación selectiva de las componentes membranales más significativas en el cilindro solamente y dejando el techo con todo el aporte membranal y flexional. Si bien no se muestran las deformadas correspondientes a los modelos MC3 y MC5, su comportamiento es similar al MC6. Se observa que las formas obtenidas presentan diferencias respecto a las deformadas observadas en el análisis no lineal (Figuras 6 y 7) en su distribución en el meridiano y en el paralelo, principalmente en los modelos en los que la relación H/D aumenta. Para el modelo MC1 se aproxima de manera aceptable la deformada en el sentido circunferencial y en el sentido del meridiano, pero en los otros casos se observan transferencias de desplazamientos en el meridiano a sectores más rígidos por mayor espesor, como en MC4 y MC6, o a sectores con sotavento, como en MC2 y MC6 en el paralelo.

\subsection{Rigidez Reducida Selectiva sobre las virolas superiores}

Al analizar estos modelos bajo presión uniforme [10] se mostró la necesidad de implementar una reducción selectiva en función del concepto de cilindro sustituto que permite controlar la transferencia de desplazamientos a zonas más rígidas por el mayor espesor que presentan las virolas inferiores. Por ello, se implementa a continuación la eliminación de las componentes meridional y torsional, solamente en las tres virolas superiores que presentan el menor espesor. Para tener en cuenta el paso de deformaciones en el paralelo hacia zonas inactivas por la presencia de menores esfuerzos, sería necesario no eliminar las componentes membranales en las zonas a sotavento. En esos sectores se producen menores desplazamientos por el pandeo localizado y deberían ser menores los esfuerzos afectando a la matriz que tiene en cuenta los esfuerzos precríticos $\left(\mathbf{N}^{\mathrm{F}}\right)$. Esta alternativa no es posible de implementar en el código ALREF, ya que la representación de los desplazamientos en el sentido circunferencial se hace a través de Series de Fourier en función del valor hallado en el meridiano y por lo tanto no se puede hacer esa reducción selectiva de las contribuciones membranales en el paralelo.

Tabla 7. Cargas críticas con rigidez reducida selectiva sobre las virolas superiores.

\begin{tabular}{|c|c|c|c|c|c|}
\cline { 2 - 6 } \multicolumn{1}{c|}{} & $\begin{array}{c}\text { Um } \\
\text { completa }\end{array}$ & \multicolumn{2}{c|}{$\begin{array}{c}\text { Um-Umss-Umst } \\
\text { (en todo el cilindro) }\end{array}$} & \multicolumn{2}{c|}{$\begin{array}{c}\text { Um-Umss-Umst } \\
\text { (en virolas superiores) }\end{array}$} \\
\cline { 2 - 6 } \multicolumn{1}{c|}{} & $\lambda^{\mathrm{C}}[\mathrm{kPa}]$ & $\lambda^{*}[\mathrm{kPa}]$ & $\eta^{*}$ & $\lambda^{* *}[\mathrm{kPa}]$ & $\eta^{* *}$ \\
\hline MC1 & 3.885 & 1.998 & 0.51 & - & - \\
\hline MC2 & 2.551 & 1.308 & 0.51 & 1.308 & 0.51 \\
\hline MC3 & 2.921 & 1.519 & 0.50 & 1.649 & 0.56 \\
\hline MC4 & 2.512 & 1.357 & 0.54 & 1.411 & 0.56 \\
\hline MC5 & 2.542 & 1.231 & 0.48 & 1.271 & 0.50 \\
\hline MC6 & 2.497 & 1.400 & 0.56 & 1.410 & 0.56 \\
\hline
\end{tabular}

La Tabla 7 presenta los valores de carga crítica hallados con reducción selectiva de las componentes membranales meridional y torsional en las tres virolas superiores solamente 
$(\lambda * *)$ y el factor de reducción ( $\left.\eta^{* *}\right)$ para cada modelo analizado, sumado a los valores obtenidos anteriormente a los efectos de comparación. Se puede observar que estos valores son levemente superiores a los hallados con la eliminación en todo el cilindro pero que conducen a coeficientes de reducción similares, con formas de modo compatibles con lo obtenido en un análisis GNIA, como se muestran en la Figura 10.
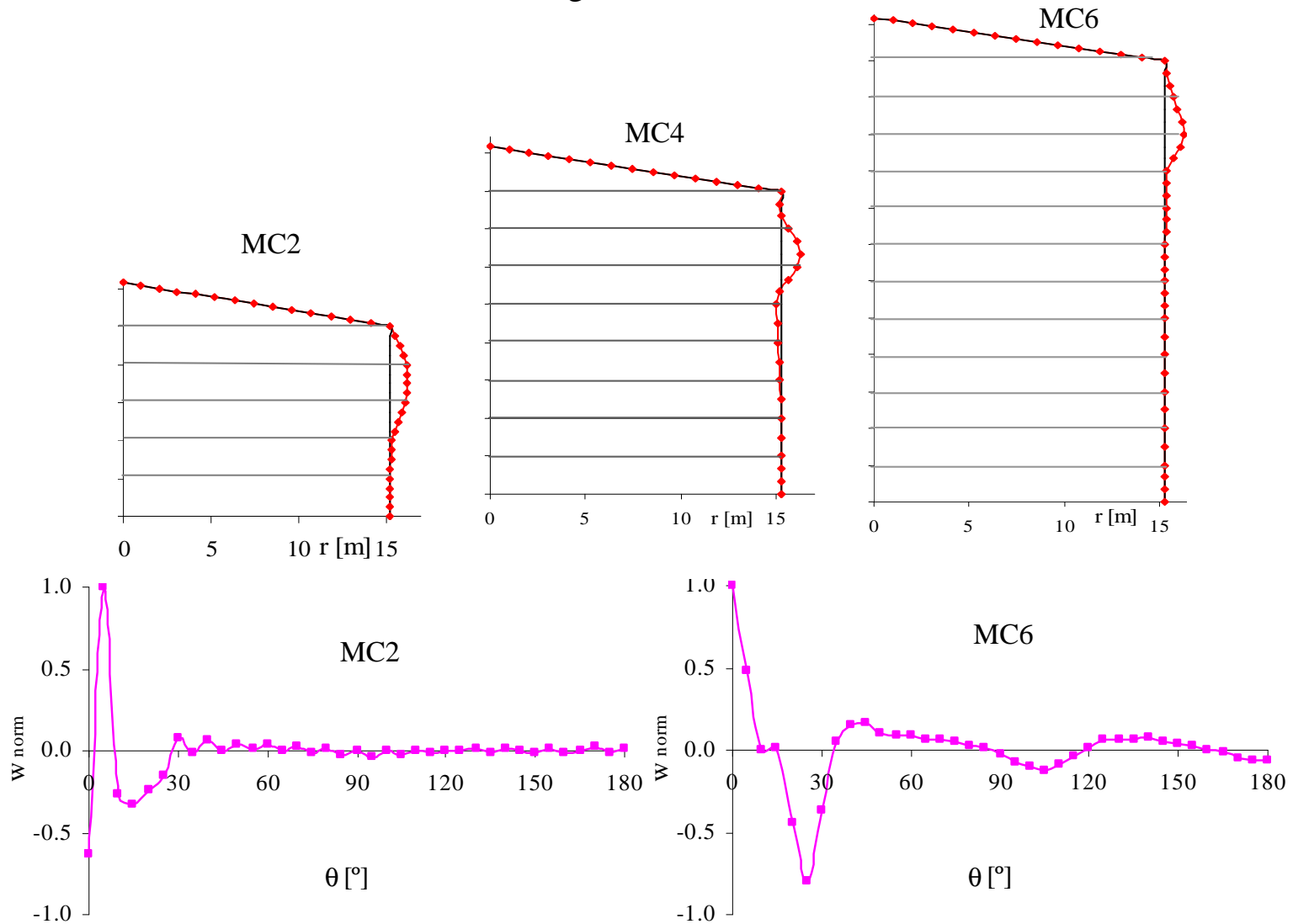

Figura 10. Formas de modo obtenidas con eliminación selectiva en las tres virolas superiores de las componentes membranales meridional y torsional.

Este planteo de retener las componentes membranales en las zonas más rígidas permite mejorar las formas de modo en el meridiano en todos los casos y corregir la distribución de desplazamientos en el paralelo, donde se obtienen formas aceptables con cargas críticas que continúan siendo un límite inferior a lo hallado en el análisis no lineal geométrico. La forma de modo para los modelos MC3 y MC5 es similar a la del modelo MC6. En estos casos la reducción selectiva de las principales componentes membranales en la zona menos rígida elimina esa transferencia de deformaciones hacia la zona con menores esfuerzos por acción del viento, aun cuando no fue posible realizar la reducción selectiva en el sentido circunferencial que sería necesaria para la eliminación selectiva del aporte membranal en la zona a sotavento.

\section{COMPARACION CON RESULTADOS EXPERIMENTALES}

En esta sección se estudian mediante la metodología de rigidez reducida un conjunto de modelos ensayados en túnel de viento presentados en el trabajo de Resinger y Greiner [15]. Se trata de un conjunto de seis modelos compuestos por una cáscara cilíndrica de espesor constante con techo plano, cuyas característica geométricas fueron indicadas en la Sección 3. 
La carga crítica $\left(\lambda^{\mathrm{C}}\right)$ para cada caso se obtiene mediante un análisis de autovalores y autovectores a través de ALREF y ABAQUS, con buen acuerdo entre ambos resultados. Estos valores se muestran en la Tabla 8 junto con las cargas críticas correspondientes a los ensayos $\left(\lambda^{\mathrm{T}}\right)$. Para todos los modelos, las componentes membranal meridional y torsional son las predominantes (Umss representa entre un 73\% y $89 \%$ de Um y Umst entre un 25 y $10 \%$ de Um, respectivamente), por esta razón se eliminan estos aportes en el problema de autovalores y autovectores. Al realizar el análisis con la metodología de rigidez reducida con una reducción homogénea de estas componentes en todo el cilindro, ya que toda la envolvente tiene el mismo espesor, se obtienen deformadas incompatibles con transferencia de deformaciones en la dirección del meridiano y del paralelo. Por ello se realiza solamente la eliminación del aporte de ambas componentes membranales en la zona superior del cilindro (la mitad superior) que presenta desplazamientos más significativos en los modos de pandeo o en los ensayos, dejando la parte inferior y el techo con el aporte completo de energía membranal. De esta manera se obtiene para cada modelo las cargas críticas $\left(\lambda^{*}\right)$ mostradas en la Tabla 8 , junto con los respectivos factores de reducción $\left(\eta^{\mathrm{T}}\right.$ y $\left.\eta^{*}\right)$ obtenidos relacionando las cargas críticas correspondientes a los ensayos y las calculadas con rigidez reducida con las provenientes del análisis de bifurcación con la energía completa. Los modos críticos asociados son compatibles con lo observado en los ensayos o en el análisis no lineal. Se puede observar que los modelos MR3 y MR5 tienen prácticamente la misma relación L/r pero MR3 tiene menor relación r/t, entre ambos modelos el que posee menor $\mathrm{r} / \mathrm{t}$ presenta una reducción menor en la carga crítica.

Tabla 8. Cargas críticas provenientes de: análisis de bifurcación $\left(\lambda^{\mathrm{C}}\right)$, ensayos en túnel de viento $\left(\lambda^{\mathrm{T}}\right)$ y de análisis de rigidez reducida en la mitad superior del cilindro $\left(\lambda^{*}\right)$.

\begin{tabular}{|c|c|c|c|c|c|c|}
\hline \multirow{2}{*}{ Modelo } & \multirow{2}{*}{$\mathrm{r} / \mathrm{t}$} & \multirow{2}{*}{$\lambda^{\mathrm{C}}\left[\mathrm{N} / \mathrm{m}^{2}\right]$} & \multicolumn{2}{|c|}{ Ensayos túnel de viento } & \multicolumn{2}{|c|}{ Rigidez Reducida } \\
\hline & & & $\lambda^{\mathrm{T}}\left[\mathrm{N} / \mathrm{m}^{2}\right]$ & $\eta^{\mathrm{T}}$ & $\lambda^{*}\left[\mathrm{~N} / \mathrm{m}^{2}\right]$ & $\eta^{*}$ \\
\hline 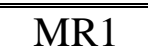 & \multirow{3}{*}{2095} & 267.9 & 229 & 0.85 & 163 & 0.61 \\
\hline MR2 & & 356.2 & 298 & 0.83 & 229 & 0.64 \\
\hline MR3 & & 534.7 & 401 & 0.75 & 349 & 0.65 \\
\hline MR4 & \multirow{3}{*}{2952} & 204.1 & 149 & 0.73 & 112 & 0.54 \\
\hline MR5 & & 259.5 & 192 & 0.74 & 129 & 0.50 \\
\hline MR6 & & 357.1 & 243 & 0.68 & 214 & 0.60 \\
\hline
\end{tabular}

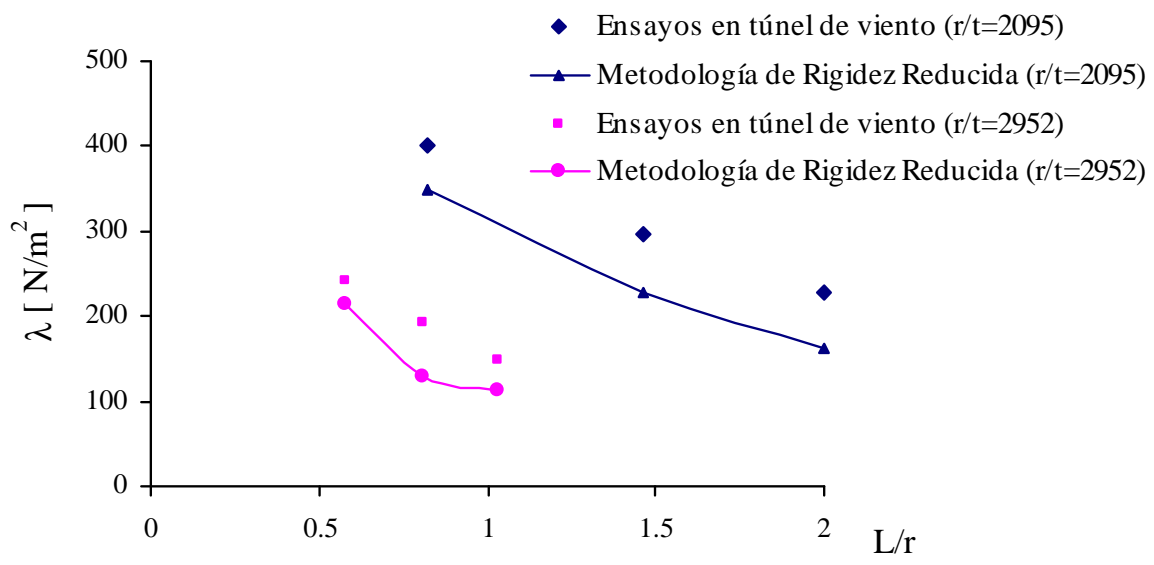

Figura 11. Cargas críticas de modelos estudiados (Resinger y Greiner [15]). 
La Figura 11 muestra las cargas críticas correspondiente a los ensayos en túnel de viento y las obtenidas por la aproximación de rigidez reducida para las relaciones de aspecto correspondientes a los modelos analizados. En la Figura 12 se presentan los factores de reducción en función del parámetro de Batdorf. En ambas figuras se puede observar que los valores hallados con la metodología de rigidez reducida representan límites inferiores a las cargas críticas correspondientes a los ensayos. Los modelos con mayor relación r/t presentan una mayor diferencia entre las cargas críticas provenientes del análisis de bifurcación y las obtenidas con la metodología de rigidez reducida, es decir los factores de reducción para MR4, MR5 y MR6 son menores. Ese comportamiento se comprueba también en los resultados de los ensayos. En la Figura 12 se observa que, en general, a medida que aumenta $\mathrm{Z}$ es mayor la diferencia entre los valores de los ensayos y los obtenidos con la metodología de rigidez reducida.

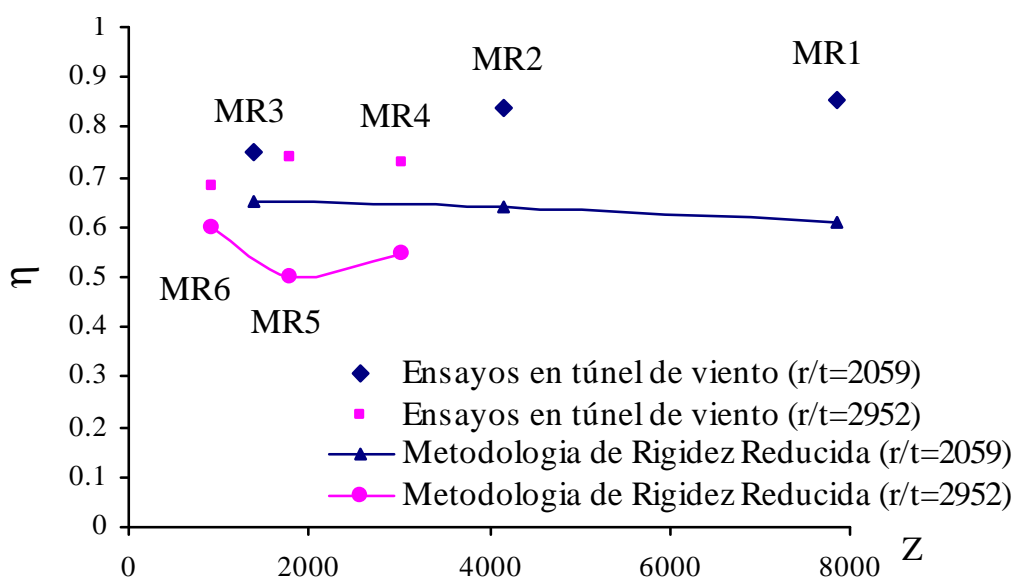

Figura 12. Factores de reducción de modelos estudiados (Resinger y Greiner [15]).

\section{CONCLUSIONES}

El método de rigidez reducida implementado en este trabajo permitió abordar la evaluación de cargas límites inferiores de las cargas críticas bajo la acción de cargas no axilsimétricas, como las de viento, para tipologías de tanques metálicos de almacenamiento de fluidos. Para ello fue necesario plantear diferentes estrategias frente a situaciones no previstas en el planteo de los desarrollos originales de Croll y colaboradores. Por un lado la existencia en la cáscara de zonas con rigideces diferentes, de manera que el sector que tiene mayor rigidez no se vería afectado por imperfecciones/interacción de modos. Por otro lado, los modos localizados como en el caso de cargas de viento que producen una zona sobre la cual no aparecen tensiones significativas, generando la dificultad de identificar sobre qué parte de la cáscara debería aplicarse la reducción de rigidez. En particular, en los casos estudiados ambas problemáticas se presenta combinadas, es decir tanques con diferentes espesores con cargas de viento.

La aplicación de esta metodología fue realizada mediante un código de propósitos específicos y arrojó resultados confiables para predecir los límites inferiores de las cargas críticas. El análisis no lineal de sensibilidad ante imperfecciones muestra resultados similares para todos los modelos de techo cónico, pero con cargas máximas mayores que las estimadas por 
el método de rigidez reducida. También se hallaron buenos resultados al comparar con valores experimentales obtenidos en túnel de viento.

Para afrontar estas problemáticas, las estrategias implementadas consistieron en: (a) Reducción homogénea, en la cual se reduce la rigidez sobre toda la superficie de la cáscara; (b) Reducción selectiva, en la cual se reduce solamente en la zona en la cual hay rigideces menores o desplazamientos en el modo. También se evaluó la alternativa de eliminar todas las componentes membranales o algunas de ellas solamente.

Al plantear una reducción homogénea en toda la cáscara se obtienen cargas críticas que representan un límite inferior pero con modos asociados muy flexibles que no aproximan bien las deformadas observadas en el análisis no lineal o en las evidencias de pandeo.

La eliminación selectiva de las componentes membranales más significativas, la meridional y torsional, en los sectores de menor rigidez permite encontrar en todos los casos estudiados límites inferiores confiables y aproximan bien las deformaciones poscríticas observadas en el análisis no lineal geométrico o en los ensayos, con formas de modos compatibles en el meridiano y aceptables en el sentido circunferencial.

Para modos localizados, debidos a viento en tanques de espesor escalonado y con techo, los factores de reducción son del orden de 0.50-0.56. Los resultados parecen indicar que la alternativa de no eliminar totalmente la energía membranal en la zona en la cual se desarrolla el modo de pandeo produce buenos resultados, tanto en la carga límite inferior como en el modo en sentido vertical y circunferencial.

Para los tanques ensayados en túnel de viento, que no presentan zonas con diferente rigidez por ser el espesor constante, el mantener algo del aporte membranal en las zonas donde el modo de pandeo presenta menores componentes en la altura del cilindro permite obtener deformadas aceptables y cargas críticas que representan límites inferiores.

\section{Agradecimientos}

Los autores agradecen la contribución de Fernando Flores quien desarrolló la versión original del código ALREF. L. A. Godoy agradece el apoyo de SECYT-UNCórdoba y CONICET en esta investigación y R. C. Jaca a SECYT-UNComahue por los subsidios para este trabajo.

\section{REFERENCIAS}

[1] Abaqus, User's Manuals, V 6.3, Hibbitt, Karlsson and Sorensen, Rhode Island, 2002.

[2] ACI-ASCE Committee 334, "Reinforced concrete cooling tower shells-practice and commentary". ACI 334, 2R. 91, American Concrete Institute. New York, 1991.

[3] API Standard 650, "Welded steel tanks for oil storage". American Petroleum Institute, Washington, D.C., 1988.

[4] Croll J. G. A., "Towards a rationally based elastic-plastic shell buckling design methodology". Thin Walled Structures. 23, 67-84, 1995.

[5] Croll J. G. A., "Towards simple estimates of shell buckling loads". Der Stahlbau, Part I, Heft 8; Part II, Heft 9. September 1975.

[6] Croll J. G. A., Ellinas, C. P., "Reduced Stiffness Axial Load Buckling of Cylinders". Int. 
J. Solids Structures. 19, 461-477, 1983.

[7] Donnell L. H., "A new theory for the buckling of thin cylinders under axial compression and bending". J Appl. Mech. Trans. ASME. 56, 795-806, 1934.

[8] Flores F. G., Godoy L. A., "Instability of shells of revolution using ALREF: Studies for wind loaded shells". Buckling of Shells in Land, in the Sea and in the Air. Elsevier Applied Science, Oxford, 213-222, 1991.

[9] Jaca R. C., Godoy L. A., Flores F. G., Croll J. G. A., "A reduced stiffness approach for the buckling of open cylindrical tanks under wind loads". Thin Walled Structures. 45, 727736, 2007.

[10] Jaca R. C., Godoy L. A., Croll J. G. A., "Reduced Stiffness Buckling Analysis of Aboveground Storage Tanks with Thickness Changes". Advances in Structural Engineering. 14, 475-487, 2011.

[11] Jaca R. C., Sosa E., Godoy L. A., "Estrategias de implementación de límites inferiores para pandeo de tanques bajo viento". Mecánica Computacional. 25, 585-604, 2006.

[12] Jaca R. C., "Limites inferiores en inestabilidad del equilibrio de láminas de tanques de pared delgada”. Tesis Doctoral. Universidad Nacional de Córdoba, 2008.

[13] Macdonald P. A., Kwok K. C. S., Holmes J. D., "Wind loads on circular storage bins and tanks: I. Point pressure measurements on isolated structures". Journal of Wind Engineering and Industrial Aerodynamics. 31, 165-188, 1988.

[14] Portela G., Godoy L. A., "Wind pressures and buckling of cylindrical steel tanks with conical roof". Journal of Construction Steel Research. 61(6), 786-807, 2005.

[15] Resinger F., Greiner R., "Buckling of wind-loaded cylindrical shells: Application to unstiffened and ring-stiffened tanks". Buckling of Shells. E.Ramm (Ed.), Springer-Verlag, Berlín, 305-331, 1982.

[16] Riks E., "An incremental approach to the solution of snapping and buckling problems". International Journal of Solids and Structures. 15, 529-551, 1979.

[17] Rotter J. M., Teng J. G., "Elastic stability of lap-jointed cylinders". Journal of Structural Engineering. ASCE, 115(3), 683-697, 1989.

[18] Rotter J. M., Chen L., Doerich C., "Buckling of cylindrical shells with stepwise variable wall thickness under uniform external pressure". Engineering Structures. 33 (12), 35703578, 2011.

[19] Rotter J. M., Schmidt H., "Buckling of Steel Shells: European Design Recommendations. $5^{\text {th }}$ Edition”. European Convention for Constructional Steelwork. Mem Martins, Portugal, 2008.

[20] Sosa E. M., Godoy L. A., "Challenges in the computation of lower-bound buckling loads for tanks under wind pressures". Thin Walled Structures. 48, 935-945, 2010.

[21] Sosa E. M., Godoy L. A., Croll J. G. A., "Computation of lower-bound buckling loads using general-purpose finite element codes". Computers and Structures. 84 (29-30), 19341945, 2006.

[22] Yamada S., Croll J. G. A., "Buckling and Post-buckling Characteristics of Pressureloaded Cylinders". Journal of Applied Mechanics. 60, 290-299, 1993.

[23] Zienkiewicz O. C., Taylor R. L., "The Finite Element Method for Solid and Structural Mechanics. Sixth Edition”. Elsevier, Oxford, U.K., 2005.

[24] Zintillis G., Croll J. G. A., "Pressure buckling of end supported shells of revolution. Engineering Structures”. 4, 222-232, 1982. 